\title{
EVALUACIÓN DEL MANEJO INTEGRADO DE PLAGAS DE Myrciaria dubia EN SUELOS NO INUNDABLES DE LA CUENCA DEL UCAYALI, PERÚ
}

\author{
José SANCHEZ-CHOY ${ }^{1}$, Carlos ABANTO-RODRIGUEZ ${ }^{2}$, Rubén CASAS-REATEGUI ${ }^{3}$ \\ 1 Instituto de Investigaciones de la Amazonía Peruana-IIAP, Programa de Investigación en Biodiversidad Amazónica \\ (PIBA), \\ 2 Programa de Investigación en Manejo Integral del Bosque y Servicios Ambientales (PROBOSQUES). Carretera Federico \\ Basadre Km 12.400, Pucallpa-Perú. Email: jsanchez@iiap.org.pe \\ 3 Universidad Nacional Intercultural de la Amazonía-UNIA, Departamento Agroforestal Acuícola. Carretera San Jose km \\ 0.5 Yarinacocha, Perú.
}

\section{RESUMEN}

El incremento de las áreas de monocultivo de camu camu está generando la proliferación de plagas y fitófagos que afectan a la producción del fruto en más de un $80 \%$. El presente trabajo evalúa la aplicación del manejo integrado de plagas (MIP) que consiste en el control cultural de podas, defoliación, y deshierbes mensuales, y control etológico con trampas amarillas y pegante entomológico así como de control biológico con aplicación de extractos acuosos de la planta biocida Paullinia clavigera. Se realiza una comparación entre este tipo de manejo y el manejo tradicional (MT) en el control de plagas de camu camu en plantaciones realizadas en suelos de altura. El experimento fue conducido mediante un diseño completo al azar (DCA), con cuatro repeticiones y cuatro plantas por unidad experimental. Las variables evaluadas fueron: porcentaje de daño en el fruto por Edessa sp y Conotrachelus dubiae, número de botones florales y frutos cuajados, y peso y diámetro del fruto. Se observó que el tratamiento con Manejo Integrado de Plagas presentó menor daño de frutos por Edessa sp (6\%) y C. dubiae (0.25\%), mayor número de frutos cuajados (497) y mayor peso del fruto a la cosecha (10.2 g) que el tratamiento con manejo tradicional. Se puede concluir que la aplicación del Manejo Integrado de Plagas reduce el ataque de plagas y mejora los componentes productivos.

PALABRAS CLAVE: camu camu, MIP, Conotrachelus dubiae, Edessa sp, biocidas

\section{ASSESSMENT OF THE INTEGRATED PEST MANAGEMENT OF Myrciaria dubia IN UNFLOODED SOILS OF THE UCAYALI BASIN, PERU}

\begin{abstract}
The increase in areas for monoculture plantations of camu camu generates the proliferation of pests and herbivores that affect fruit production by more than $80 \%$. This study evaluates the application of an integrated pest management technique which consist of applying cultural control of pruning and defoliation and monthly weeding, ethological control with yellow and entomological glue-traps, Biological control using aqueous extracts of the biocide plant Paullinia clavigera. We compared this management technique with the traditional management (MT) in the control of pests of camu camu plantations in upland soils. The experiment used a completely randomized design (CRD) with four replicates and four plants per experimental unit. The variables evaluated were: percentage of fruit damage by Edessa sp and Conotrachelus dubiae, number of flower buds and set fruits, and weight and diameter of the fruit. We observed that the treatment with integrated pest management presented significantly less fruit damage by Edessa sp (6\%) and C. dubiae (0.25\%); greater number of set fruits (497) and increased fruit weight at harvest (10.2 g) than the treatment with traditional management. We concluded that the application of integrated pest management reduces pests and improved productive components.
\end{abstract}

KEYWORDS: camu camu, integrated pest management, Conotrachelus dubiae. 


\section{INTRODUCCIÓN}

El camu camu arbustivo Myrciaria dubia H.B.K Mc Vaugh, es una especie frutícola de la Amazonía con ventajas comparativas, debido a un elevado contenido de ácido ascórbico que puede alcanzar los $3.500 \mathrm{mg} / 100 \mathrm{~g}$ de pulpa (Pinedo, 2010), superando 40 veces el contenido total de la naranja y 55 veces el contenido del limón (Yuyama et al., 2002). Posee, además, calcio, fósforo, tiamina, riboflamina y niacina. (TCA, 1996). Todas estas características hacen que tenga un gran interés en el mercado local, nacional e internacional. En los últimos 20 años este cultivo ha recibido una mayor atención, por lo que su domesticación ha tenido avances importantes, tales como la ampliación del cultivo a zonas no inundables de la Amazonía (Zapata \& Dufour, 1993).

Las áreas de cultivo de Camu camu en la región Ucayali se han incrementado en los últimos años, contando en la actualidad con 5.000 has sembradas, de las cuales, 400 has se encuentran en producción, con un rendimiento promedio de fruta en suelos de altura y restinga de $7,85 \mathrm{~kg}_{\text {planta }}{ }^{-1}$ y $8,72 \mathrm{~kg}$ planta $^{-1}$ respectivamente (Pezo, 2011). Este incremento de las áreas de producción está generando la proliferación de plagas importantes que están afectando hasta en un $80 \%$ el rendimiento del cultivo. Se han registrado hasta 70 especies de insectos fitófagos que dañan los frutos del camu camu (Delgado \& Couturier, 2004). Entre estas plagas podemos considerar como de importancia económica al picudo del camu camu (Conotrachelus dubiae), al chinche del camu camu (Edessa sp.) y al pegador de hojas (Tuthillia cognata), especies que proliferan tanto en ecosistemas inundables (restinga) como en suelos no inundables.

Los frutos del camu camu se destinan principalmente para el consumo humano, por lo que hay serias restricciones en el uso de agroquímicos para el manejo del cultivo. Frente a esta situación es necesario proponer estudios que permitan usar la biodiversidad vegetal existente, como las plantas biocidas, combinadas con otras técnicas, para lograr un control orgánico en el manejo integrado de plagas (MIP). La agricultura orgánica favorece el control biológico de las plagas, siendo este un posible camino para reducir el uso de pesticidas en la agricultura moderna (Puech et al., 2014).

El MIP debe ser flexible y no regirse por protocolos y debe implementar, al menos, dos componentes de manejo compatibles entre sí, ya que un enfoque multilateral de técnicas contribuye a la estabilidad del agro ecosistema. Así mismo, un MIP exitoso deberá enfocar sus prioridades en el agricultor, ya que es este agricultor el que acepta el cambio, pone en riesgo sus recursos, evalúa los beneficios de la nueva tecnología y decide su adopción (Cisneros, 2010).

El objetivo de este trabajo fue evaluar los resultados en el control de plagas del fruto del camu camu en plantaciones de suelos de altura aplicando Manejo Integrado de Plagas (MIP) y manejo tradicional de plagas (MT).

\section{MATERIALES Y MÉTODOS}

El ensayo se realizó en la estación experimental Dale E. Bandy del IIAP-Ucayali, en el distrito de Yarinacocha, Región Ucayali. La estación experimental está geográficamente ubicada a $8^{\circ} 22^{\prime} 31^{\prime \prime}$ de la latitud sur y $74^{\circ} 34^{\prime} 35^{\prime \prime}$ de longitud oeste, a una altitud de $154 \mathrm{msnm}$, en un área de 648 $\mathrm{m}^{2}$, en una plantación de 10 años de edad. La plantación se diseñó con un distanciamiento de $3 \mathrm{~m}$ entre filas x $3 \mathrm{~m}$ entre plantas (1 111 plantas $\mathrm{ha}^{-1}$ ), por un periodo de 7 meses, desde el 15 de marzo al 15 de octubre del 2013. La clasificación ecológica de la zona de estudio corresponde al ecosistema de Bosque tropical Semi-Siempre Verde Estacional (Cochrane 1982). La temperatura promedio durante el experimento fue de $26^{\circ} \mathrm{C}$, con máximas de 31,1 ${ }^{\circ} \mathrm{C}$ y mínimas de $22,5^{\circ} \mathrm{C}$. La precipitación pluvial fue de $736 \mathrm{~mm}$, alcanzando la mínima durante el mes de setiembre, con $52 \mathrm{~mm}$ y la máxima durante el mes de octubre, con $176 \mathrm{~mm}$. El suelo del área experimental pertenece a la categoría ultisol de baja fertilidad, con un porcentaje de materia orgánica de 2,7\%, nitrógeno mineral con $97 \mathrm{kgha}^{-1}$, fósforo, como $\mathrm{P}_{2} \mathrm{O}_{5}$, con $498 \mathrm{kha}^{-1}$, potasio, como $\mathrm{K}_{2} \mathrm{O}, 217$ $\mathrm{kgha}^{-1}$, una relación entre cationes $\mathrm{Ca} / \mathrm{mg}$ deficiente en Magnesio, con pH 5,18, muy fuertemente ácido. Se utilizó un diseño experimental completo al azar con cuatro repeticiones y cuatro plantas por unidad experimental.

En primer lugar se llevó a cabo un manejo integrado de plagas (MIP), a través de la aplicación de varios tipos de control:

Control cultural, realizando podas de renovación, defoliación de las plantas de camu camu, deshierbo mensual por 7 meses y recojo de frutos caídos. La poda se realizó cuando las plantas de camu camu entraron al estado vegetativo (latencia), con la finalidad de modificar su proceso biológico, acelerar su desarrollo vegetativo y desequilibrar los ciclos biológicos de algunas plagas.

Control etológico, utilizando trampas amarillas $(30 \times 60 \mathrm{~cm})$ con un pegante entomológico. Las trampas se colocaron en una rama a una altura de $1,30 \mathrm{~m}$, en sentido contrario al viento, con un 
intervalo de 30 días a una densidad de 1 trampa por $144 \mathrm{~m}^{2}$. Se realizaron 5 instalaciones antes y después de la floración. Durante la floración no se utilizaron tramas amarillas para evitar la captura de insectos polinizadores.

Control biológico, aplicando extractos acuosos por cocción de plantas biocidas de Sachayoco (Paullinia clavigera) al 5\% de concentración. El extracto se aplicó 15 días después de la floración, es decir, desde la fase 2 (clavito) hasta la fase 8 (fruto maduro), por un periodo de 50 días, tal como lo describe Inga et al. (2001). Se realizaron 4 aplicaciones cada 10 días, hasta los 205 días del periodo productivo. El extracto madre vegetal fue cocinado en agua en una proporción de $100 \mathrm{~g}$ de material de hojas secas en $1.000 \mathrm{ml}$ de agua, por 20 minutos de ebullición; el extracto madre filtrado se conservó en condiciones de refrigeración a $5{ }^{\circ} \mathrm{C}$, protegido de la luz directa del sol, en frascos de vidrio ámbar con boca ancha y forrada con papel aluminio. Posteriormente se diluyó para las aplicaciones en campo en mochila fumigadora de $20 \mathrm{~L}$.

El segundo tratamiento consistió en el manejo tradicional (MT) de una parcela y consistió en hacer deshierbes con motocultivadora cada 60 días por tres oportunidades. No se realizaron podas, ni recojo de frutos caídos.

Los parámetros evaluados fueron el porcentaje de daño en el fruto por Edessa sp. y Conotrachelus dubiae, tanto en frutos en el estado pintón maduro como en aquellos en estado maduro; el número de botones florales y frutos cuajados, seleccionando, para ello, cuatro ramas por planta, ubicadas en los cuatro puntos cardinales; el conteo de botones florales luego de la polinización por 10 días.

A los 180 días después de la defoliación (DDD) se contó el número de frutos en estado verde. Para determinar el peso y tamaño del fruto, se realizó el conteo y medición transversal con un vernier digital, de 200 frutos recogidos de ramas seleccionadas al azar en estadio pintón maduro a los 205 DDD. Los datos fueron sometidos a un análisis de varianza (ANVA) y los promedios fueron comparados mediante la prueba de Tukey al $95 \%$ de confiabilidad, los análisis fueron realizados con el programa estadístico SAS (1999).

\section{RESULTADOS Y DISCUSIÓN}

\section{Porcentaje de daño en fruto por Edessa sp y Conotrachelus dubiae}

En la Tabla 1 se observan diferencias significativas en el análisis de varianza entre los tratamientos en estudio con una $\mathrm{F}$ de 10.7 y 34.71 para el porcentaje de daño por Edessa sp. y Conotrachelus dubiae respectivamente. La prueba de Tukey mostró diferencias entre las medias de los tratamientos, observándose mayor daño con MT.

La combinación de diferentes métodos de control en el tratamiento MIP, tales como la poda y defoliación y la eliminación de ritidomas de las ramas y troncos donde se esconde el picudo Conotrachelus dubiae durante el día, probablemente rompió el ciclo de vida de la plaga en el campo. Así mismo, la poda mejoró la iluminación de la parcela, produciendo mayor calor sobre el terreno y generando condiciones adversas para la plaga (Abanto, 2014). De otro lado, la menor presencia de Conotrachelus dubiae se debió a la ruptura de su ciclo de mayor incidencia en suelos no indudables (octubre a diciembre), periodo que coincide con la floración y fructificación natural de las plantas de camu camu (Perez, 2008).

De otro lado, con el control cultural de deshierbes mensuales, se eliminaron los hospederos del chinche de la especie Edessa sp, plaga que, durante la fase vegetativa de latencia del camu camu, se alimenta y hospeda en malezas como sachahuaca (Baccharis floribunda) y gramíneas como Paspalum notatum. Al respecto, Pinedo et al. (2001), sostiene que el refugio de este chinche se encuentra en las plantas invasoras.

Es notorio que el control etológico con trampas de color amarillo también ha reducido el número de adultos del género Edessa sp.

Finalmente, la aplicación de una planta biocida como Paullinia clavigera, ha permitido repeler el ataque de insectos al fruto, debido a la presencia de saponinas, fenoles, flavonoides, cumarinas y quinonas, tal como menciona Pérez et al. (2008).

Se ha observado un mayor ataque de chinches en los estadios verdes de la fruta, mientras que el número de ataques a las frutas maduras fueron protagonizados por el picudo de camu camu.

El MT no remueve hojas viejas del campo, tampoco elimina fuentes de infestación, al realizar solo una limpieza del campo al inicio de la campaña y luego cada dos meses.

\section{Numero de botones florales y frutos cuajados}

En la Tabla 1 se observa el número de botones florales desde los 120 días después de la defoliación (DDD), hasta los 130 DDD, no encontrándose diferencias estadísticas en el análisis de varianza entre los tratamientos MIP y MT. Sin embargo, existen diferencias significativas para el número de frutos verdes a los 180 DDD. La prueba de Tukey presentó significancia del tratamiento con MIP, con 
un $80 \%$ superior en el número de frutos cosechados.

El número de botones florales reportados entre MIP y MT no coinciden con lo reportado por Abanto et al. (2014), en donde aseguran que con la aplicación de la poda de fructificación y defoliación se encontró una mayor capacidad productiva de los botones florales. Probablemente esto último fue debido al nivel de poda realizado. El número de frutos cuajados, sin embargo, si coincide con lo reportado por los mismos autores y Quijada et al. (2009), coincidiendo en que la poda induce a la floración y formación de frutos en la guayaba. Es muy probable que lo que esté ocurriendo sea una pérdida de la dominancia apical de las hojas viejas que no permiten la aparición de brotes por la presencia de hormonas naturales en la parte aérea.

De otro lado, la aplicación del MIP podría estar protegiendo y permitiendo que estos cuajen, evitando así la caída por picaduras de Edessa sp. Durante el llenado del fruto, como lo reportan Farro \& Pinedo (2010), Edessa sp. es causante de un $9.15 \%$ de la caída de los frutos, siendo Conotrachelus dubiae el responsable de la caída de un $0.12 \%$ de los frutos. El $90.7 \%$ de las caídas es provocado por factores fisiológicos, nutritivos o atmosféricos no determinados. Así mismo, se menciona que los factores ambientales de temperatura mayores a $27.7{ }^{\circ} \mathrm{C}$, influyen directamente en la caída del fruto. Las épocas de sequía provocan también una mayor caída del fruto.

\section{Peso $(\mathrm{g})$ y diámetro del fruto $(\mathrm{mm})$}

En la Tabla 1 se observan diferencias estadísticas en el análisis de varianza entre los tratamientos realizados para la variable peso del fruto, observándose un incremento del $20 \%$ con respecto al tratamiento con MT. No se encontró diferencias en el diámetro del fruto entre los tratamientos.

Una mejor respuesta de la planta con la aplicación de MIP durante el llenado de frutos, probablemente se deba a una menor competencia por los escasos nutrientes del suelo, debido al control de las malezas presentes y a la menor presencia de plagas. Así mismo, el menor peso reportado con el tratamiento MT puede deberse a la presencia de la plaga Edessa sp. que pica el fruto durante su llenado, causando oxidación y desintegración del ácido ascórbico, como lo describe Delgado \& Courturier (2004). De otro lado, las características fenológicas del diámetro del fruto, probablemente no son afectadas por la condiciones externas, debido a una característica genética dominante de la especie camu camu.

Las ventajas en el uso del MIP no se pueden ignorar, ya que son económica y ambientalmente favorables, coincidiendo plenamente con lo mencionado por Freitas et al. (2011).

\section{CONCLUSIONES}

El Manejo Integrado de Plagas (MIP) fue más eficiente en el control de plagas del fruto del camu camu en suelos de altura que el manejo tradicional de plagas (MT), mejorando el proceso productivo de este frutal.

\section{AGRADECIMIENTOS}

Se agradece la colaboración del técnico Rusber Tapullima del programa PIBA en Ucayali y de los bachilleres Alan Tello y Jack Soberon, por el apoyo en la colecta de datos de campo.

Tabla 1. Resultados para las variables evaluadas en plantación de camu camu con aplicación de Manejo Integrado de Plagas (MIP) y Manejo Tradicional (MT) en suelos no inundables de Pucallpa, Perú.

\begin{tabular}{|c|c|c|c|c|c|c|c|c|}
\hline \multirow[t]{2}{*}{ Tamaño } & \multicolumn{2}{|c|}{$\begin{array}{c}\% \\
\text { Daño }\end{array}$} & \multicolumn{3}{|c|}{$\begin{array}{l}N^{\circ} \text { Botones Florales a días } \\
\text { después de la defoliación (DDD) }\end{array}$} & \multirow{2}{*}{$\begin{array}{c}\begin{array}{c}\mathbf{N}^{\circ} \text { Frutos } \\
\text { Cuajados }\end{array} \\
\begin{array}{c}180 \\
\text { DDD }\end{array}\end{array}$} & \multirow{2}{*}{$\begin{array}{c}\text { Diámetro } \\
\text { fruto } \\
(\mathrm{mm})\end{array}$} & \multirow{2}{*}{$\begin{array}{c}\text { Peso } \\
\text { Fruto } \\
\text { (g) }\end{array}$} \\
\hline & Edessa sp & $\begin{array}{c}\text { Conotrachelus } \\
\text { dubidae }\end{array}$ & $\begin{array}{l}120 \\
D D D\end{array}$ & $\begin{array}{l}125 \\
D D D\end{array}$ & $\begin{array}{l}130 \\
D D D\end{array}$ & & & \\
\hline MIP & $6.0 \mathrm{~b}$ & $0.25 b$ & 406 a & $558 \mathrm{a}$ & $716 a$ & $497 a$ & $25.7 \mathrm{a}$ & $10.2 \mathrm{a}$ \\
\hline MT & $12.7 \mathrm{a}$ & $2.5 \mathrm{a}$ & $142 \mathrm{a}$ & 348 a & $418 \mathrm{a}$ & $98 \mathrm{~b}$ & $24.2 \mathrm{a}$ & $8.02 \mathrm{~b}$ \\
\hline$\overline{F F}$ & 10.7 & 34.71 & 1.61 & 0.48 & 2.09 & 7.62 & 3.78 & 9.82 \\
\hline $\operatorname{Pr}>F$ & $0.016^{*}$ & $0.001 * *$ & $0.25 \mathrm{~ns}$ & $0.39 \mathrm{~ns}$ & $0.19 \mathrm{~ns}$ & $0.03 *$ & $0.1 \mathrm{~ns}$ & $0.02 *$ \\
\hline C.V (\%) & 31 & 39 & 107 & 71 & 51 & 68 & 4.3 & 10.6 \\
\hline
\end{tabular}

Letras iguales en la misma columna no presentan significancia a la prueba de medias de Tukey con $\mathrm{P}<0.05$.

F: Valor F de Fisher del ANVA; $\operatorname{Pr}>$ F: Probabilidad mayor a Fisher del ANVA, C.V: Coeficiente de Variación.

ns: no significativo * Significativo a $\mathrm{P}<0.05 *$ *altamente significativo $\mathrm{P}<0.001$ 


\section{BIBLIOGRAFÍA CITADA}

Abanto Rodríguez Carlos, Mario Pinedo Panduro, Ricardo bardales Lozano, Edvan Alvez Chagas. Efecto de la poda de fructificación y defoliación en el proceso productivo de Camu camu en la región Ucayali-Perú. Folia Amazónica, 23(1)2014:17-24

Cisneros, Fausto. 2010. Control de plagas agrícolas, fascículo 13 el manejo integrado de plagas. En: Control de plagas MIP. P. $1-35$ (http://hortintl.cals.ncsu.edu/sites/default/files/a rticles/Control de Plagas Agricolas MIP Ene 2010.pdf). Acceso: 21/10/2014

Cochrane, T. T. 1982. Caracterización Agroecológica para el desarrollo de pasturas en suelos ácidos de América Tropical. En: Toledo J.M. (Ed). Manual para la evaluación de Agronómica. Red Internacional de Evaluación de Pastos Tropicales (RIEPT). Calí, Colombia, CIAT. p. 23-24

Delgado, C.; Courturier, G. 2004. Manejo de insectos plagas en la Amazonia: Su aplicación en Camu camu. IIAP - IRD. 147pp.

Farro, Sonia; Pinedo, Mario. 2010. Posibles factores que producen la caída de fruto de Myrciaria dubia (HBK) Mc Vaugh, "Camu camu" durante la fenología reproductiva de la colección "cinco cuencas" en el centro experimental San Miguel IIAP, Loreto, Perú. Scientia Agropecuaria 1: 117 $-123$.

Freitas, A.; Batistela,M.; Freitas Bueno, R.; FrançaNeto,J.; Naime Nishikawa, M.; Filho, A. 2011. Effects of integrated pest management, biological control and prophylactic use of insecticides on the management and sustainability of soybean. Crop Protection, 30: 937-945

Inga, H., Pinedo, M., Delgado C., Linares C., Mejía K. 2001. Fenología Reproductiva de Myrciaria dubia McVAUGH (H.B.K.) Camu camu. Folia Amazónica, 12 (1-2):99-106

Pérez, D., Iannacone, J., Tueros, T. 2008. Toxicidad de Paullinia clavigera Schltdl. (Sapindaceae) y del Chondrodendron tomentosum Ruiz et Pav. (Menispermaceae) sobre el piojo saltador del Camu camu Tuthillia cognata Hodkinson (Hemíptera: Psyllidae). Gayana Botánica ISSN. 65(2):145-152.
Pérez, D.; Iannacone, J. 2008. Ciclo biológico, comportamiento y censo del picudo del Camu camu, Conotrachelus dubiae O'Brien 1995 (Coleoptera: Curculionidae) en Pucallpa, Perú. Acta Amazônica, 38(1): 145 - 152

Pezo, E. 2011. Identificación de las especies silvestres de insectos polinizadores y su influencia en la producción de Camu camu (Myrciaria dubia H.B.K. Mc Vaugh) en un entisol de Pucallpa. Tesis pre-grado, Universidad Nacional de Ucayali. Facultad de Ciencias Agropecuarias, Pucallpa, Perú. 70pp.

Pinedo, M.; Rita, R.; Rengifo, E.; Delgado, C.; Villacres, J.; Gonzáles, A.; Herminio, I.; López, A.; Ferronay, R.; Linares, C. 2001.Sistema de producción de Camu camu en restinga. IIAP. Programa de Ecosistemas Terrestres. Proyecto Bioexport Camu camu, Perú. 141pp.

Pinedo, M. 2010. Camu camu (Myrciaria dubia, Myrtaceae) Aportes para su aprovechamiento sostenible en la Amazonia Peruana. Instituto de Investigaciones de la Amazonia Peruana, Iquitos. $135 \mathrm{pp}$.

Puech, C.; Baudry, J.; Joannon, A.; Poggi, S; Aviron, S. 2014. Organic vs. conventional farming dichotomy: Does it make sensefor natural enemies?. Agriculture, Ecosystems and Environment 194 (2014) 48-57

Quijada, O.; Ramírez, R.; Corzo, P. 1999. Efecto de la poda y la cianamida hidrogenada sobre la brotación, fructificación, producción y calidad de frutos del guayabo (Psidium guajava L.) en el municipio de Mara del estado de Zulia, Revista de la Facultad de Agronomia (LUZ), 16:276-290

SAS INSTITUTE INC. 1999. SAS. Cary, NC, USA. Tratado de Cooperación Amazónica. 1996. El Cultivo del Camu camu (Myrciaria dubia H.B.K. Mc Vaugh) en la Amazonía Peruana. $84 \mathrm{pp}$

Yuyama, K.; Aguiar, J. P. L.; Yuyama, L. K. O. 2002. Camu-camu: un fruto fantastico como fonte de vitamina C. Acta Amazônica 32: 169-174.

Zapata, S.M.; Dufour, J.P. 1993. Myrciaria dubia (HBK) Mc Vaugh: Chemical composition of fruit.

Recibido: 20 de febrero del 2015

Aceptado para publicación: 10 de abril del 2015 
